\title{
A Source Routing Interference-Aware Multi-Path Routing Protocol for Mobile Ad Hoc Networks
}

\author{
Phu Hung Le
}

\begin{abstract}
In the literature, several Interference-Aware Multi-path Protocols have been introduced for Mobile Ad hoc NETworks (MANETs) but they are either not highly efficient or have the computational complexity of NP-hard. To tackle the problems, in this paper, we propose a novel Source Routing Interference-Aware Multi-path Protocol (SR-IA-MPOLSR) for mobile ad hoc networks. Our protocol uses source routing mechanism, parallel transmission via the minimal interference paths and adapts quickly to the change of topology in MANETs. The other strong point of SR-IAMPOLSR is that it finds paths with the computational complexity in polynomial time instead of NP-hard of other protocols. We compare our protocol to two typical protocols AOMDV (multipath protocol) and DSR in terms of packet delivery fraction, end-to-end delay, and routing overhead when the RTS/CTS mechanism is alternatively enabled and disabled. From simulation results, we show that SR-IA-MPOLSR significantly improves the network performance. We also prove the the network performance is reduced when RTS/CTS mechanism is used.
\end{abstract}

Index Terms-Mobile ad hoc networks, multipath, routing protocol, OLSR, interference.

\section{INTRODUCTION}

Mobile ad hoc networks consist of a collection of wireless mobile nodes that move freely and self-configure without a preexisting communication infrastructure.

In MANETs, because the nodes move freely and its topology is dynamic, the routing protocols for fixed networks are not suitable. Hence, many routing protocols have been proposed to adapt to the rapid change of topology in MANETs, for example, single path protocols such as AODV [1], DSR [2], OLSR [3], and OLSR-Feedback [4] or multi-path protocols such as AOMDV [5], SR-MPOLSR [6], SMR [7] and MPOLSR [8], etc. However, most of them chose the minimum hop-count routes. The choice can lead to significant reduction of the network performance because communication quality via the chosen links is not good. Thus, link quality is a essential problem to be considered.

In [9], the authors specified the interference level by twohop interference region. In [10], link's interference degree is an average interference degree of two nodes forming the link. The authors of [11] estimated the residual bandwidth in transmission range. Some protocols such as [12], [13], and

Manuscript received February 1, 2014; revised May 16, 2014.

Phu Hung Le is with Banking Academy of Vietnam (e-mail: Lephuhung7@yahoo.com)
[14] evaluate the link quality based on the Expected Transmission Count (ETX) metric [15] but only considered the inner-link interference, and ignored the impact of the outside nodes as well as the interference level by the geographic distance between nodes. Furthermore, these metrics do not perform well in mobile ad hoc networks because they do not react sufficiently rapidly demonstrated in [16].

To repair weak points above, a few interference-aware multi-path protocols were proposed for mobile ad hoc networks. Ying-Hong Wang et al., [17] propose IMRP but it is not high efficient. The protocol of W. Wei et al., [18] is a heuristic protocol that is only suitable for static or slow moving nodes. Kamal Jain et al., [19] used the conflict graph to find paths. However, the computational complexity of this solution is NP-hard that significantly reduces the computational speed. In practice, this solution cannot be used.

To overcome these limitations, we propose a new interference-aware multi-path protocol called Source Routing Interference-Aware Multi-path protocol (SR-IAMPOLSR) that is very simple to implement. Its computational complexity is only in polynomial time, but high efficient for mobile ad hoc networks. SR-IA-MPOLSR is built by determining an interference region of a link consisting of all nodes that can interfere with the link and evaluating the interference level of a link based on the number of nodes impacting on the link and the geographic distance between nodes. Then, we model a MANET as a weighted directed graph with the weight of each arc equal to the link interference level, we can find the least interference paths thanks to Dijkstra's algorithm (the computational complexity of $\mathrm{O}(\mathrm{n} 2)$ ).

To increase throughput and reduce end-to-end delay and routing overhead, SR-IA-MPOLSR uses source routing technique (the route is put in the header of packet) to simultaneously transmit packets through the least interference node-disjoint paths (these paths are nodedisjoint to eliminate the bottleneck nodes). To demonstrate the efficiency of the protocol, we compare SR-IA-MPOLSR to AOMDV and DSR in a mobile environment when the RTS/CTS mechanism is alternatively turned on and turned off.

The RTS/CTS mechanism is to restrict the collision caused by the hidden terminals. However, we show that the RTS/CTS mechanism is not suitable for ad hoc networks.

This paper is divided into four sections. In Section II, we introduce the detail structure of the protocol SR-IAMPOLSR. Section III compares the protocols SR-IAMPOLSR, AOMDV and DSR. Our results are summarized in Section IV. 


\section{Source Routing Interference-Aware Multi- PATH PROTOCOL}

\section{A. Topology Discovery}

We assume that a node knows its position by using GPS.

SR-IA-MPOLSR consists of all components in HELLO and Topology Control (TC) messages of OLSR. It also does:

Each node adds its carrier sensing range and position to HELLO message. Nodes record the information in the HELLO message.

Each node that creates TC messages adds the carrier sensing range, the neighbor information and the position of it, the carrier sensing range, the neighbor information and the position of nodes in MPR selector set to TC messages. When the nodes in the network receive a TC message, they save the information in the message to calculate the interference level of links.

Like OLSR, HELLO messages are periodically sent to its neighbors while TC messages are sent to the entire network.

\section{B. Definition of Link Interference}

In a MANET, when a node transmits data, all nodes within the carrier sensing range are interfered. The level of the interference of a node depends on the distance from the transmitting node to received node.

In the literature, there are many different definitions of interference in a MANET. One of the famous interference definitions is of M. Burkhart et al., [20]. [20] definites coverage of a link e $(\operatorname{Cov}(e))$ between two nodes $u, v(\mathrm{e}=(u$, $v)$ ) is the cardinality of nodes covered by the disks induced by $\mathrm{u}$ and $v$ :

$$
\begin{gathered}
\operatorname{Cov}(e)=\mid\{w \text { in } V \mid \text { w is covered by } D(u,|u, v|)\} U \\
\{w \text { in } V \mid \text { w is covered by } D(v,|v, u|)\}
\end{gathered}
$$

Here, $V$ is the set of all nodes of network, $|u, v|$ is Euclidean distance between $u$ and $v . D(u,|u, v|)$ and $D(v, \mid v$, $u \mid)$ are circles that the centers are $u$ and $v$, the radii are $|u, v|$ and $|v, u|$, respectively.

However, this definition has some disadvantages: 1) it does not considers nodes that are outside of $\mathrm{D}(u,|u, v|)$ or $D(v,|v, u|)$ but they still interfere with the link $e=(u, v)$ because interference radius of a node $u$ or $\mathrm{v}$ is much larger than $|\mathrm{u}, \mathrm{v}|$ proved in [21]; 2) in [20], $\operatorname{Cov}(\mathrm{e})$ depends on the distance between $u$ and $v$. This is not exact because any node within the carrier sensing range of $u$ or $v$ can impact on the link e no matter how the distance between $u$ and $v$ is.

To repair above disadvantages, we define an interference region of a link $e=(u, v)$ (unidirectional or bidirectional) as follows:

$$
\begin{aligned}
I(e)=\mid & \{w \in V \mid w \text { is covered by } D(u, \mathrm{Rcsu})\} U \\
& \{w \in V \mid \text { w is covered by } D(v, \mathrm{R} c s v)\} \mid
\end{aligned}
$$

Here, $V$ is the set of all nodes of network. Rcsu and Rcsv are carrier sensing range of $\mathrm{u}$ and $\mathrm{v}$, respectively. $D(u, \mathrm{Rcsu})$ and $D(v, \mathrm{Rcsv})$ are circles that the centers are $u$ and $v$, the radii are Rcsu and Rcsv, respectively.

Our definition repaired the weak points in definition of $M$. Burkhart et al., because it includes all nodes that can interfere with the link and also overcome the weak point ii) indicated above in [20] because the cardinality of nodes interfering with a link does not depend on the distance between two nodes creating the link.

\section{Estimation of Link Interference}

We measure interference of a link based on the geographic distance between nodes in a MANET and the cardinality of nodes that can affect the link. We also divide the whole interference region of a link into smaller interference regions to exactly evaluate the interference of a link and a path. The estimation of link interference will be more precise as we divide the interference area of a link into smaller areas. However, it increases the calculation complexity.

We divided the interference region into one or two smaller regions but the protocol is not efficient as expected as there is no differentiation or a slight differentiation of the interference level by distance between nodes.

Thus, in this paper the interference region is divided into four regions. This choice is a compromise between the precision and the computational complexity. The interference regions are following.

The whole interference region of a link $e=(u, v)$ can be considered as in our definition in B part. We divide the circle with center $\mathrm{u}$ and radius $R c s u$ into four circles by $R_{1 u}$, $R_{2 u}, R_{3 u}$ and $R_{4 u}$ and divide the circle with center $\mathrm{v}$ and radius Rcsv into four circles by $R_{1 v}, R_{2 v}, R_{3 v}$ and $R_{4 v}$ (Fig. $1)$.

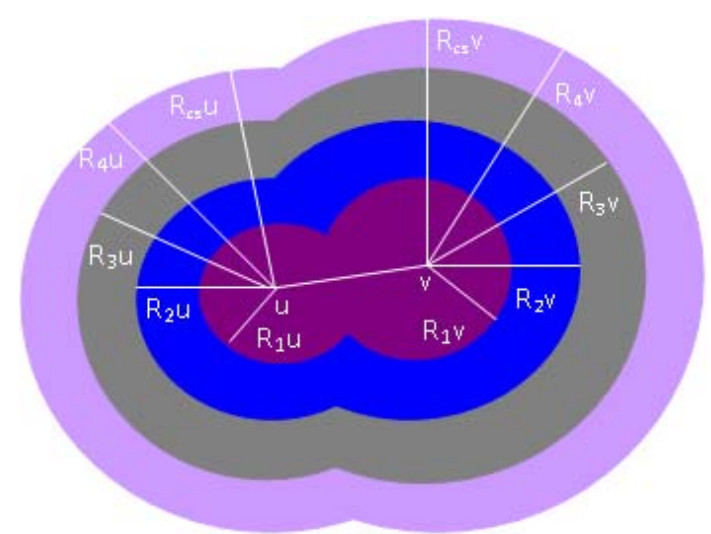

Fig. 1. Illustration of radii of interference.

where,

$$
\begin{aligned}
& R_{1 u}=1 / 4 \mathrm{Rcsu}, R_{1 v}=1 / 4 \mathrm{Rcsv} \\
& R_{2 u}=2 / 4 \mathrm{Rcsu}, R_{2 u}=2 / 4 \mathrm{Rcsv} \\
& R_{3 u}=3 / 4 \mathrm{Rcsu}, R_{3 v}=3 / 4 \mathrm{Rcsv} \\
& R_{4 u}=R c s u, R_{4 v}=R c s v
\end{aligned}
$$

Four zones are specified below.

$$
\begin{aligned}
& \text { Zone }(0)=\emptyset \text { (empty) } \\
& \text { Zone(i) }=\left\{w \in V \mid \text { w is covered by } D\left(u, R i_{u}\right)\right\} U
\end{aligned}
$$

$\left\{w \in V \mid\right.$ w is covered by $\left.D\left(v, R i_{v}\right)\right\} \backslash$ Zone $(i-1), i \in[1,4]$

For each zone, we assign an interference weight which represents the interference level that a node in this zone causes to the considered link. The Zone (1) (violet) has the highest interference level. The Zone (2) (blue) has a higher interference level than the Zone (3) (gray). The interference 
level of Zone (4) (lavender) is smallest.

If the weight of interference of zone (1) is 1 , the weight of interference of zone (2), zone (3) and zone (4) are $\alpha, \beta, V$ respectively $(V<\beta<\alpha<1)$. We can calculate the interference of a link $e=(u, v)$ in a MANET as follows:

$$
I(e)=n_{1}+\alpha . n_{2}+\beta . n_{3}+n_{4}
$$

where $n_{1}, n_{2}, n_{3}$ and $\mathrm{n} 4$ are the number of nodes in zone (1), zone (2), zone (3) and zone (4), respectively. Parameters $\alpha$, $\beta$ and $V$ are determined as follows. According to [21], in Two-Ray Ground path loss model, the receiving power $\left(P_{r}\right)$ of a signal from a sender $\mathrm{d}$ meters away can be modeled as Eq. (2).

$$
P_{r}=P_{t} G_{t} G_{r} h_{t}^{2} h_{r}^{2} / d^{k}
$$

In Eq. (2), $G_{t}$ and $G_{r}$ are antenna gains of transmitter and receiver, respectively. $P_{t}$ is the transmission power of a sender node. $h_{t}$ and $h_{r}$ are the heights of both antennas respectively. Here, we assume that a MANET is homogeneous, that is all the radio parameters are identical at each node. We can apply the formula (2) to $u$ or $v$.

$$
\left.0.5^{k}{ }^{\alpha}=P_{t} G_{t} G_{r} h_{t} h_{r} / R_{2 u}{ }^{k}\right) /\left(P_{t} G_{t} G_{r} h_{t} h_{r} / R_{1 u}{ }^{k}\right)=R_{1 u}{ }^{k} / R_{2 u}{ }^{k}=
$$$$
\beta=\left(P_{t} G_{t} G_{r} h_{t} h_{r} / R_{3 u}{ }^{k}\right) /\left(P_{t} G_{t} G_{r} h_{t} h_{r} / R_{1 u}{ }^{k}\right)=R_{1 u}{ }^{k} / R_{3 \underline{\underline{u}}}=
$$
$0.33^{k}$ $0.25^{k}$

$V=\left(P_{t} G_{t} G_{r} h_{t} h_{r} / R_{4 u}{ }^{k}\right) /\left(P_{t} G_{t} G_{r} h_{t} h_{r} / R_{1 u}{ }^{k}\right)=R_{1 u}{ }^{k} / R_{4 u}{ }^{k}=$

We assume that common path loss model used in wireless networks is the open space path loss which has $k$ as 2 . Therefore, $\alpha=0.25, \beta=0.11, V=0.06$ and

$$
I(e)=n_{1}+0.25 n_{2}+0.11 n_{3}+0.06 n_{4}
$$

Based on the formula (3), we can calculate interference of a path $P$ that consists of links $e_{1} e_{2}, \ldots, e_{n}$

$$
I(P)=I\left(e_{1}\right)+I\left(e_{2}\right)+\ldots+I\left(e_{n}\right)
$$

\section{SR-IA-MPOLSR Protocol Design}

We develop SR-IA-MPOLSR as follows.

1) Specifying $n_{1}, n_{2}, n_{3}, n_{4}$

According to the formula (3), the interference of a link $e$ $=(u, v)$ in a MANET is

$$
I(e)=n_{1}+0.25 n_{2}+0.11 n_{3}+0.06 n_{4}
$$

Each node of a MANET has a co-ordinate $(x, y)$. The coordinate of a node is defined by writing a program in NS-2. In reality, we can use GPS to specify the co-ordinate of a node. If the co-ordinate of $u, v$ is $\left(x_{1}, y_{1}\right),\left(x_{2}, y_{2}\right)$, respectively, then distance between $u$ and $v$ is

$$
\sqrt{\left(x_{1}-x_{2}\right)^{2}+\left(y_{1}-y_{2}\right)^{2}}
$$

The formula (4) is used to calculate the distances between $\mathrm{u}$ and all other nodes in a MANET. After comparing those distances to $R_{1 u}, R_{1 v}, R_{2 u}, R_{2 v}, R_{3 u}, R_{3 v}, R_{4 u}, R_{4 v}$ we will have the number of nodes in zone (1), zone (2), zone (3), and zone (4) of the link e.

\section{2) Modelling a MANET as a weighted directed graph}

A MANET can be considered as a weighted directed graph (Fig. 2) where nodes of a MANET are vertices of the graph and the arcs of the graph are any connection between two nodes. The status of each arc is defined by information in HELLO and TC messages. The weight of each arc is the interference level of the corresponding link.

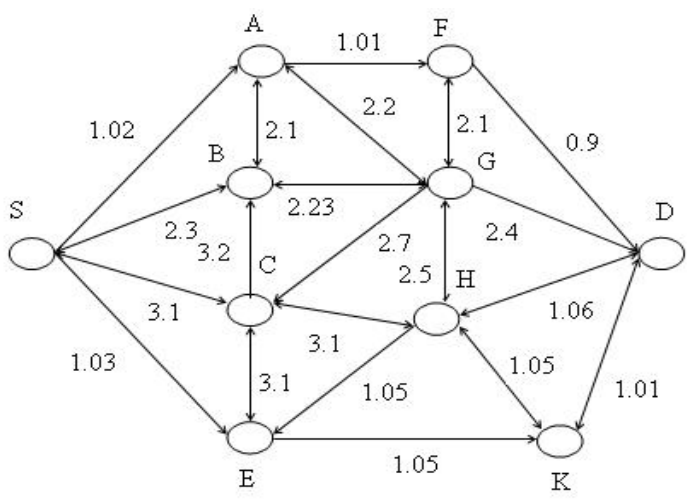

Fig. 2. Illustration of SR-IA-MPOLSR.

\section{3) Algorithm of node-disjoint multi-path}

Node-disjoint multi-path: the paths have only common source and destination.

To find node-disjoint paths, we execute on the same weighted directed graph as following steps :

Step 1: The minimum interference path is based on Dijkstra's algorithm.

Step 2: To get the second minimum interference path from the source to the destination, Dijkstra's algorithm is applied once more while avoiding all nodes between the source and the destination along the path found in the step 1.

Step 3: Dijkstra's algorithm is repeated for a number of times $k(k=3, \ldots, n)$ while avoiding all nodes between the source and the destination along the paths found in the previous steps to find k-minimum interference path.

For example: In the Fig. 2, we illustrate an example for MANET that is considered as a weighted directed graph. The weight of each arc is set on the arc.

Applying the Dijsktra's algorithm at the first time for this weighted graph with the source $\mathrm{S}$ and the destination $\mathrm{D}$ we get the minimum interference path S-A-F-D that has the path interference value of 3.02 .

Using the Dijsktra's algorithm once more, we get the second minimum interference path S-E-K-D with the path interference value of 3.09. We continue to run the Dijsktra's algorithm and find the third path S-B-G-D with the interference value of 6.93 .

\section{4) Source-routing transmission of SR-IA-MPOLSR}

Unlike Unlike our previous protocols [22], [23], [24], and other protocols, SR-IA-MPOLSR chooses source routing machanism that puts the route into the header of packet to simultaneously transmit packets through the least interference node-disjoint paths. These paths are nodedisjoint to eliminate the bottleneck nodes and decrease delay.

\section{Performance Evaluation}

\section{A. Simulation Environment}

Our protocols are implemented in NS2. The simulations are realized with 55 nodes. Each node has a transmission 
range of 160 meters and a carrier sensing range of 400 meters placed randomly within the area of $550 \mathrm{~m} \times 550 \mathrm{~m}$. The nodes move with the speed from $1 \mathrm{~m} / \mathrm{s}$ to $30 \mathrm{~m} / \mathrm{s}$. The pause time is set to $15 \mathrm{~s}$. The distributed coordination function (DCF) of IEEE 802.11 for wireless LANs is used as the MAC layer. Traffic source is CBR and channel capacity is 11 Mbps. Constant Bit Rate (CBR) keeps at $100 \mathrm{Kbps}$. The Two-Ray Ground and the Random Waypoint models are also used in our simulations. The simulation time is 120 seconds. Each data column represents an average of two runs with different random mobility scenarios. The RTS/CTS mechanism is alternatively enabled and disabled.

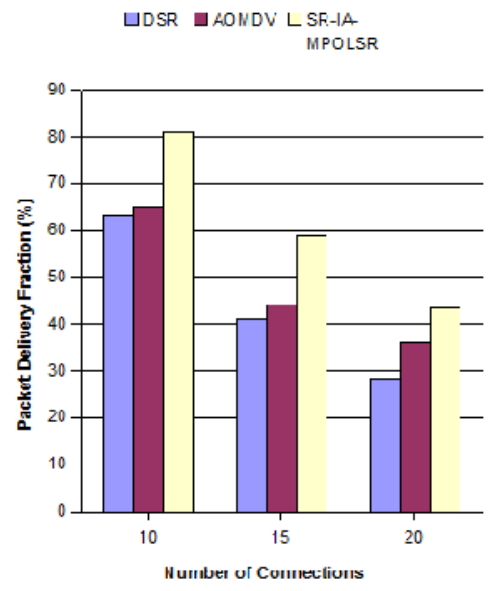

Fig. 3. Packet delivery fraction with RTS/CTS

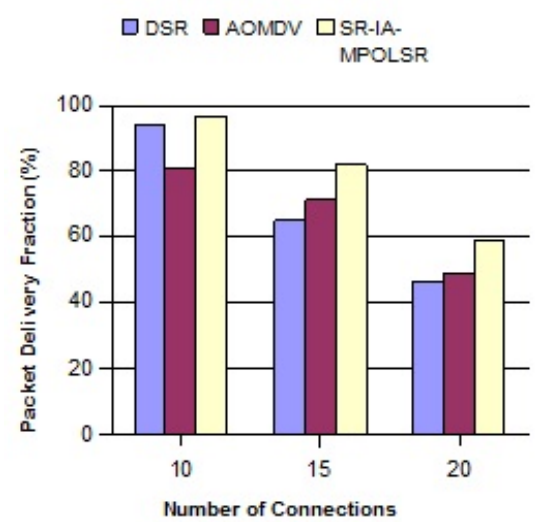

Fig. 4. Packet delivery fraction without RTS/CTS.

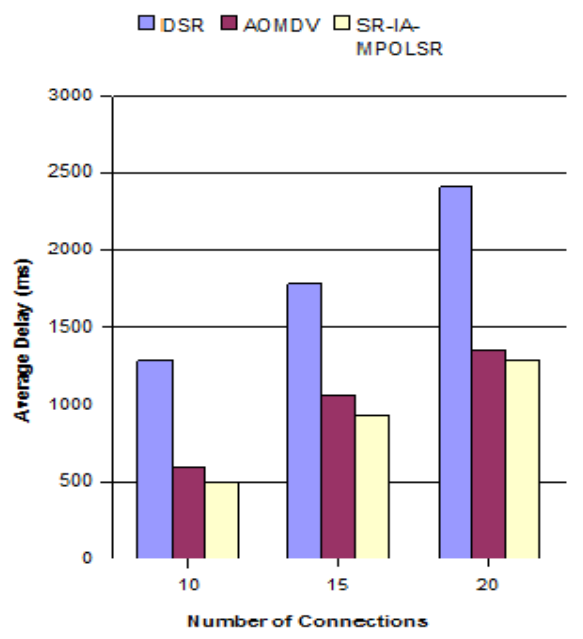

Fig. 5. Average delay with Rts/Cts.

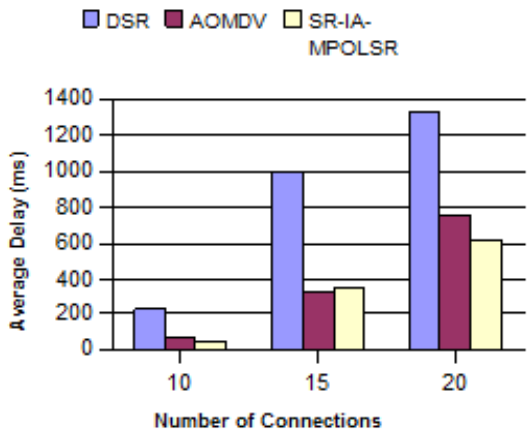

Fig. 6. Average delay without RTS/CTS. $\square D S R \square$ AOMDV $\square$ SR-IAMPOLSR

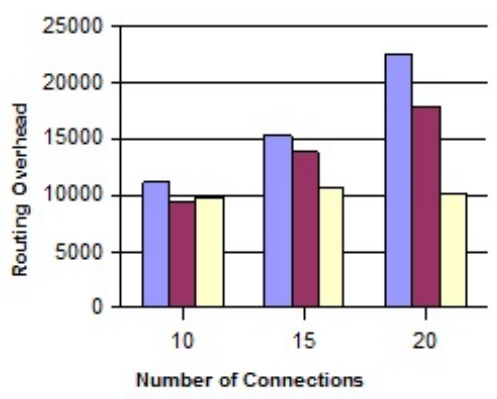

Fig. 7. Routing overhead with RTS/CTS. $\square D S R \quad \square$ AOMDV $\square$ SR-IAMPOLSR

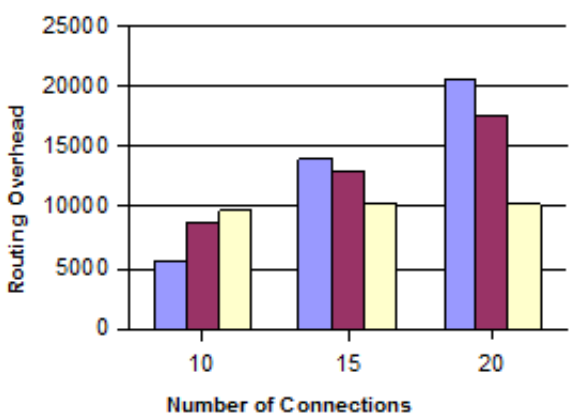

Fig. 8. Routing overhead without Rts/Cts.

\section{B. Simulation Results}

We compare three protocols SR-IA-MPOLSR, AOMDV and DSR in terms of i) Packet delivery fraction (PDF)-the ratio of the received data packets at the destination to those sent by the source; ii) Average end-to-end delay-the average time that packets travel from the source to the destination; iii) Routing overhead- the average total number of packet controls transmitted at each node.

Fig. 3 and Fig. 4 show that in a quite dense network and a mobile environment, the PDF of the three protocols decreases when the number of source-destination connections increases. SR-IA-MPOLSR outperforms other protocols in terms of PDF. Fig. 3 shows that in terms of PDF, with 10 connections, SR-IA-MPOLSR is $15.95 \%$ and $17.82 \%$ higher than AOMDV and DSR, respectively because SR-IA-MPOLSR has lower interference. In Fig. 4, when RTS/CTS are turned off, the PDFs of all protocols increase much compared to those of them when RTS/CTS is used. It is about $31 \%$ for DSR, $16 \%$ for the other protocols with 10 connections. However, at 10 and 20 connections, the difference between SR-IA-MPOLSR and AOMDV is 
now $16.2 \%$ and $10 \%$, an increase of $0.25 \%$ and $3 \%$ for SRIA-MPOLSR, respectively.

The delay of each protocol without using RTS/CTS is lower than it's delay with using RTS/CTS. With 10 sourcedestination pairs, the delays of SR-IA-MPOLSR, AOMDV, and DSR reduce around 10, 9 and 5.5 times compared to the delays of them, respectively when RTS/CTS is used as seen in Fig. 5 and Fig. 6. Also in terms of delay, with 10 connections and turning off RTS/CTS, SR-IA-MPOLSR improves about $35 \%$ and $80 \%$ compared to AOMDV and DSR, respectively. This is because SR-IA-MPOLSR has less contention. DSR has always the highest delay. It increases a lot when the number of connections increases.

In Fig. 7 and Fig. 8, routing overhead of two reactive protocols increases when the number of source-destination pairs increases, especially DSR. DSR and AOMDV have a reduction of routing overhead when RTS/CTS is not used but they are still 2 and 1.7 times more than SR-IA-MPOLSR, respectively at 20 connections since the mobile environment leads to the increase of link breakages. SR-IA-MPOLSR is usually lower and more stable than DSR and AOMDV in term of routing overhead because of the efficiency of Multipoint Relays (MPRs) and characteristic of proactive protocol.

\section{CONCLUSION}

A big challenge to design routing protocols for mobile ad hoc networks is rapid reaction and efficiency. Some existing interference-aware multi-path protocols are not high efficient or very high computational complexity (NP-hard).

This paper proposed a new Source Routing InterferenceAware Multi-path protocol (SR-IA-MPOLSR) for mobile ad hoc networks based on our definition of interference and a formula of interference. SR-IA-MPOLSR has a reasonably computational complexity (polynomial time) and a high efficiency. This was proved by comparing to the prominent protocols AOMDV and DSR in a mobile environment when the RTS/CTS mechanism is alternatively turned on and turned off. Indeed, the proposed protocol significantly improves the performance in the most important metrics such as packet delivery fraction, end-to-end delay, and routing overhead.

Based on our simulation results, we also showed that the RTS/CTS mechanism significantly reduces the network performance.

The link interference evaluation is an important and difficult problem.

Interference of a link includes inter-link and outer-link interference. We studied interference outside of a link. The problem of inter-link interference was considered in literature but only with static network. We will study the problem of inter-link interference in mobility environment to improve the performance of our protocols.

\section{REFERENCES}

[1] C. E. Perkins and E. M. Royer, "Ad-Hoc on demand distance vector routing," IEEE Work-Shop on Mobile Computing Systems and Applications (WMCSA), New Orleans, 1999, vol. 90, no. 100.

[2] D. B. Johnson, D. A. Maltz, and J. Broch, "DSR: The dynamic source routing protocol for multi-hop wireless ad hoc networks," Ad Hoc Networking, 2001, pp. 139-172.
[3] T. Clausen and P. Jacquet, IETF Request for Comments: 3626 , Optimized Link State Routing Protocol OLSR, October 2003.

[4] Masimum. [Online]. Available: http://masimum.inf.um.es/fjrm/

[5] M. K. Marina and S. R. Das, "On-demand multipath distance vector routing for ad hoc networks," Wireless Communications and Mobile Computing, vol. 6, pp. 969-988.

[6] X. Zhou, Y. Lu, and B. Xi, "A novel routing protocol for ad hoc sensor networks using multiple disjoint paths," in Proc. 2nd International Conference on Broad, vol. 2, pp. 944-948, 2005.

[7] S. J. Lee and M. Gerla, "Split multi-path routing with maximally disjoint paths in ad hoc networks," IEEE ICC 2001, 2001, pp. 32013205 .

[8] J. Yi, E. Cizeron, S. Hamma, B. Parrein, and P. Lesage, "Implementation of multipath and multiple description coding in OLSR," Networking and Internet Architecture, vol. 3, 2009.

[9] D. Q. Nguyen and P. Minet, "Interference-aware QoS OLSR for mobile ad-hoc network routing," in Proc. SNPD-SAWN '05, pp. 428435, 2005.

[10] X. Zhang, Q. Liu, D. Shi, Y. Z. Liu, and X. Yu, "An Average link interference-aware routing protocol for mobile ad hoc networks," in Proc. ICWMC'07, p. 10, 2007.

[11] L. Ma, Q. Zhang, F. An, and X. Cheng, "A dynamic interference aware routing. protocol for IEEE 802.11-based. mobile ad hoc networks," in Proc. MSN, LNCS, vol. 3, pp. 2223-2234, 2005.

[12] R. Draves, J. Padhye, and B. Zill, "Routing in multi-radio, multi-hop wireless mesh networks," in Proc. Mobile Computing and Networking, Philadelphia, PA, USA, 2004, pp. 114 - 128.

[13] I. Sheriff and E. B. Royer, "Multipath selection in multi-radio mesh networks," in Proc. Broad-Multi-radio Mesh Networks, pp. 1-11, 2006.

[14] R. Langar, N. Bouabdallah, R. Boutaba, and G. Pujolle, "Interferer link-aware routing in wireless mesh networks," in Proc. IEEE ICC, pp. 1-6, 2010.

[15] D. D. Couto, D. Aguayo, J. Bicket, and R. Morris, "A highthroughput path metric for multi-hop wireless routing," in Proc. MobiCom, San Diego, CA, vol. 11, no. 4, 2003.

[16] R. Draves, J. Padhye, and B. Zill, "Comparison of routing metrics for static multi-hop wireless networks,"in Proc. Sigcomm '04, vol. 34, no. 4, 2004.

[17] Y. H. Wang and H. Z. Lin, "Multipath QoS routing with interference provision in ad hoc wireless network," Journal of Information Science and Engineering, pp. 1325-1338, 2006.

[18] W. Wei and A. Zakhor, "Interference aware multi-path selection for video streaming in wireless MANETs networks," IEEE Transactions on Circuits and Systems for Video Technology, pp. 110-122, 2009.

[19] K. Jain, J. Padhye, V. Padmanabhan, and L. Qiu, "Impact of interference on multi-hop wireless network performance," in Proc. the 9th MobiCom, vol. 11, no. 4, pp, 471-487, 2005.

[20] M. Burkhart, P. Rickenbach, R. Wattenhofer, and A. Zollinger, "Does topology control reduce interference?" in Proc. MobiHoc, pp. 9-19, 2004.

[21] K. Xu, M. Gerla, and S. Bae, "Effectiveness of RTS/CTS handshake in IEEE 802.11 based ad hoc networks," Journal of Ad Hoc Networks, 2003, vol. 1, no. 1, pp. 107-123.

[22] P. H. Le, G. Pujolle, and T. M. T. Nguyen, "An interference-aware multi-path routing protocol for mobile ad HOC network," in Proc. 8th IEEE International Conference on Networking, Sensing and Control (ICNSC 2011), Delft, the Netherlands, April 2011, IEEEXplore, pp. $503-507$.

[23] P. H. Le and G. Pujolle, "A Link-disjoint interference-aware multipath routing protocol for mobile ad hoc network," in Proc. the International Conference on Digital Information and Communication Technology and its Applications(DICTAP 2011), Dijon, France, June 2011, Springer LNCS, pp. 649-661.

[24] P. H. Le, "A Performance evaluation of multi-path routing protocols for mobile ad hoc network," in Proc. the 10th IEEE/IFIP International Conference on Embedded and Ubiquitous Computing, Paphos, Cyprus, pp.484-491, 2012.

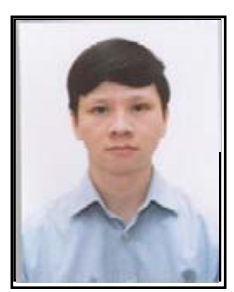

Phu Hung Le is with Banking Academy of Vietnam. $\mathrm{He}$ received the Ph.D degree in computer science from Pierre Marie Curie University (Paris 6), France. He also obtained B.S and M.S degrees in computer science from Vietnam National University. Phu Hung Le is an author of many articles and is a reviewer for many international conferences and journals. 